\title{
Application of Information System in Human Resource Management
}

\author{
Xiao Han \\ Hubei University Of Economics
}

\begin{abstract}
With the development of China's economy, more and more Chinese companies have appeared. And with the emphasis on the role of human, these companies are paying more and more attention to human resource management. The development of informatization has brought many benefits to the human resources management of enterprises. This article focuses on the concept and advantages of human resource information systems, and how to scientifically establish a human resource management system.
\end{abstract}

Keywords: human resource; information system; informationization

Author's Informarion: Xiao Han (July 4, 1998), female, Xiangyang, Hubei, China, undergraduate in department of information management and information system, Hubei University Of Economics.

DOI: $10.36012 / \mathrm{ems} . v 1 \mathrm{i} 1.959$

\section{Introduction}

\subsection{Research Background}

A $\mathrm{s}$ the arrival of the knowledge economy and information society has become a consensus, enterprises, an important subject in social activities, are also developing along with social changes. The development of any enterprise is inseparable from resources, whether tangible or intangible, and Human Resources, as one of the important components, has also changed its role in enterprise development. At present, Human Resources has become companies' prime resource, and human resource management has changed its role. It has become a consensus to establish the competitive advantages of enterprises through the management and development of human resources. The function of human resource management has also changed from the function of traditional personnel management to the role of strategic human resource management. In order to successfully complete its role transfer and better realize the strategic partner functions of human resource management, more and more companies have shifted their functions through organizational restructuring, process reengineering, business outsourcing, corporate culture shaping, and informationization.

Viewing from the current circumstance, there are already extraordinarily mature products in the field of enterprise information technology, such as financial management software, production management software, and supply chain management software. The e- mergence of these software, on the one hand, has injected new management concepts into enterprise management; on the other hand, it has also caused profound changes in the way companies work. Comprehensive informationization will be the trend of future enterprise development. Under such circumstances, human resource management informatization is bound to be a newcomer in the field of informatization in the future.

With the continuous development of China's economy, the development of the domestic human resource management information system has gradually matured. To meet the various functional requirements, common management information systems are feasible.

With reference to the survey conducted by Chen Meina, Qin Yan, and Zhao Yuxin in 2010, 62.82\% of the companies participating in the survey were IT companies with a higher level of informatization. The scale of enterprises mainly concentrates on large and medium-sized enterprises with 1,000 to 5,000 employees and SMEs with less than 500 employees.

Surveys have shown that "e-HR" has become a more prevalent trend in HR functions. $75 \%$ of enterprises have realized the informationization of human resources. Modern enterprises have increasingly shifted traditional manual business to information software platforms for processing, which has freed human resource management departments from tedious personnel affairs, payment issues, attendance, etc. Moreover, it has transferred more energy to enterprises in terms 
of talent develo pment strategy, which has become a strategic partner of enterprise management.

However, although some companies have already established human resource management information systems, e-HR, as a "double-edged sword", has exposed many problems in specific applications. For instance, advanced management concepts are inconsistent with the corporate culture, advanced system functions have not been brought into practice, and long-term planning is in shortage.

The current level of human resource management informatization in China's enterprises is not high, and it is urgent to develop in the future. Therefore, it is necessary to study the related issues of human resource management informationization.

\subsection{Research Purposes}

The purpose of this article is to explore how to combine human resource management with informatization. Integrating human resource management with information technology is an important research area for personnel and experts who have been engaged in human resource management in the past decade. The main purpose of the automation and informationization of human resources work is to liberate HR managers from the heavy transactional routine work, and the core is to guide the work towards a more effective enterprise-level human resource development plan. In order to greatly improve the comprehensive benefits of human resource management, experts have applied information technology and automation technology on a large scale. From recruiting to dismissing, the human resource management information system has strengthened the management of employees' life cycle. Information technology and human resource management theory should be used as the foundation to standardize human resource management processes, and special attention should be paid to the promotion of selecting, training, and human capital planning.

From foreign research materials and related reports, it is found that the HR electronification is one of the important trends in the development of human resources at present and in the future. The word "e-HR" is a less strict term. Human resource management information system refers to the integration of information technology and human resource management into the management practice of the organization through the establishment of an information platform, making it suitable for the specific needs of enterprises, capable of handling normative and exceptional, universal, special, relatively simple, and contextually complex structural tools. E-HR is actually a human resource management information system based on Internet / Intranet. This is a new human resource management system and employee communication method developed on the Internet platform. "E-HR" includes all parts of human resources that can be technologically innovative, and it can automate and electronicize personnel management processes. In addition to the traditional human resource information system, "e-HR" has a greater significance in that it changes the way employees communicate with the enterprise, thereby making the "people" in the enterprise more subjective. The human resource management of Chinese enterprises is gradually moving towards "e-HR". E-HR places high requirements on the company's hardware environment, the quality of its employees, and the company's management level. This is a major constraint on development at this stage.

To conclude, China is in a "transition period" and the market economy is not fully developed, so the management concept needs to be further updated, and enterprises have a long way to go to adapt to the development of the network economy. Faced with this new concept of e-HR, the emergence and existence of various problems are inevitable, and the road to e-human resource management is still waiting for exploration. In this situation, it is very necessary for this paper to explore the path of human resource electronification and the solution suitable for the informatization of human resource management of local enterprises.

\section{Related Concepts of Human Resource Management Information System}

In this section, two concepts will be introduced: Human Resource Management and Human Resource Management Information System.

\subsection{Human Resource Management}

For Human Resource Management, two parts will be included: the concept as well as the development and problems of human resource management.

2.1.1 The concept of human resource management

The concept of "human resources" was proposed by Peter F. Drucker, a famous contemporary management scientist, in his book "Practices in Management" in 1954. For the whole society, it refers to the collection of labor capacity (human physical strength, knowl- 
edge, and skills) possessed by the entire population; while for an enterprise, it only includes what it owns or controls and can be used for a certain period of time. The labor capacity of the entire population, which constitutes an individual and overall relationship with social human resources, is a concrete manifestation of the latter's value. Human resource management refers to the continuous acquisition of human resources and the rational allocation of human resources in a planned manner in accordance with the requirements of corporate development strategies, through a series of processes such as recruitment, training, assigning, assessment, motivation, and adjustment of employees in the enterprise. This process is aimed at maintaining and motivating employees' loyalty and enthusiasm for the organization, exerting the potential of them, creating value for the enterprise, and ensuring the realization of corporate strategic goals. These are a series of human resource policies and corresponding management activities of the enterprise. By forecasting the organization's human resource requirements and making human demand plans, recruiting and selecting personnel and conducting effective organization, assessing performance, paying compensation and effective incentives, combining organizational and individual needs for effective development, an enterprise can achieve optimal organizational performance.

2.1.2 Development and Problems of Human Resource Management

The traditional HR department is often called the "Personnel Department" or "Employee Relations Department", which mainly plays the role of administrative expert and employee motivator. This department takes various issues related to employees seriously, ensures that employees are paid corresponding compensation. Additionally, it manages employees' labor contracts, and avoids relevant legal issues. HR departments need to ensure that employee-related issues do not interfere with the operation of the company's products or services. This kind of human resource management is basically a passive response, that is, only when human resource issues directly affect the business development of the enterprise, HR departments will be concerned. Although this situation still exists in many companies, others are convinced that human resource management is very important to business success. They have expanded the role of human resource management to changing agents and strategic partners, while traditional functions are expended by new technologies.

With the application of technology in many administrative management matters, such as managing personnel information records of employees, allowing employees to obtain training, benefits and other various human resource items, enrolling employees and registering and so on., the administrative role of human resource management began to fade away.

The development of new technologies such as the Internet has weakened the role of human resource management in maintaining personnel records and allowed employees to obtain self-service.

The role of the HR department in the development of management practices and strategic partners has been strengthened. One of the most comprehensive research shows that "human resource management is transforming from a specialized, independent function to a wider range of company capabilities. In this kind of company capacity building, human resource managers and line managers foster a partnership to gain competitive advantages and achieve the company's overall business goals." HR managers are increasingly being absorbed into high-level committees that can influence the company's decision-making. These HR managers' report directly to the CEO, the president or the board of directors, and put forward proposals to solve various business problems.

Today's enterprises face many challenges, such as the challenges of the new economy, globalization, stakeholders, and high-performance work systems. In order to make enterprises stand out in the fierce competition, the requirements for human resource management are higher. Many problems can no longer be solved in the traditional way. We must use more scientific and advanced methods to deal with them. Human resource information system and electronic human resource management (e-HR) came into being. Although they appear only to solve the problem of high-performance work systems, to this day or even later, human resource management information systems have or will help enterprises to meet more challenges in a larger scope.

\subsection{Human Resource Management Information System}

Three parts are included in this part: concept, development history and necessity of Human Resource Management Information System. 
2.2.1 Concept of Human Resource Management Information System

Human Resource Management Information System (HRMIS) refers to the development of enterprise human resource management theory. By establishing an information platform, information technology and human resource management theory are integrated into the organization's management practice. Designed to make it suitable for the specific needs of enterprises and departments, it helps companies define and optimize business processes for human resource management, improve work efficiency as well as service quality, and provide information-based support to become a personalized and professional platform. A good HMRIS solution should be a system that provides the best support for all aspects of human resource management, including not only the general functions such as human resource planning, talent recruitment, employee training and development management, performance management, employees salary and benefits management, as well as basic personnel data information (employee information management, contract management, attendance management, vacation management and departure management), but also provide information statistical analysis and report output functions, which can timely and dynamically reflect the status of corporate human resources, for corporate human resource strategy provides effective decision support. With the development of technology, the self-service and internal communication service must also be gradually added.

With the development and the application of e-commerce, a human resource management mode e-HR (electronic human resource management) based on mature network technology and enterprise information platform emerges at the historic moment. E-HR is different from HRMIS, which is an information management model that includes Intranet / Internet and establishes standard human resource management processes to achieve the sharing of human resource management information and the effective integration of enterprise resources. Different from traditional human resource management, it is based on the idea of "total human resource management". By using information technology such as the internal local area network and database, the human resource management concept and management process of the enterprise are solidified through the system to achieve norms and stan- dards and process work platform. On this platform, various roles in the human resource management ecosystem can use the system to work and communicate.

2.2.2 Development History of Human Resource Management Information System

The human resource management system has its own development history. At present, a large number of domestic human resource management information systems are emerging, some of which are provided by foreign manufacturers, and some are provided by domestic manufacturers. In general, the development of human resource management systems has gone through the following four stages:

1) 1970s-basic calculation tool stage: Currently, the human resource management system is just in its infancy. It is mainly a tool for automatic salary calculation, which can only realize automatic calculation after salary data input, cannot retain historical salary data and has no data analysis function.

2) 1980s-the era of salary and personnel systems: At this time, the human resource management system realized the preservation and management of historical salary data and had functions of report generation and data analysis. However, the main functions are still staff management and salary calculation, which do not cover other businesses in human resource management.

3) 1990s-The era of human resource management systems: At that time, the human resource management system used a centralized database to integrate almost all HR-related data such as salary and benefits, recruitment, personal career design, language training, job management, performance management, job description, personal information, and the historical data, which formed an integrated information base, and automated and collaborative work was achieved through standardized and perfect business processes.

4) After the 21 st century-the era of human resource management information (e-HR): Currently, the human resource management system integrates various types of information about human resources, increases the accuracy, and intensifies the real-time nature of the data. It can also make human resource planning according to the strategic goals of the organization, realizing the reasonable allocation of human re sources, performing human cost analysis, realizing the analysis and monitoring of corporate human resources 
for individual projects, and providing decision support. Additionally, this system interacts with other modules within the enterprise to improve departmental collaboration.

2.2.3 Necessity of Human Resource Management Information System

1) Reduce business management costs: The emergence of the system frees the HR department from a lot of complicated affairs. For example, the entry and modification of batch data will consume a lot of time and energy of the HR department, but it will not contribute to the profit growth of the enterprise. After the system is applied, the basic personnel and salary information can be imported and modified in batches, which reduces the repetitive work of the HR department. When the scale of the enterprise is expanded, the enterprise does not need to increase the staffing. Instead, the increased amount of information can be completed through the system. Therefore, for the HR department, labor costs are reduced.

2) Improve the efficiency of human resource management: Information technology is widely used in human resource management. It develops special systems and procedures in salary and benefit calculation, recruitment, training, attendance, performance evaluation, and job description. HR managers can transfer the data storage and analysis calculations to the corresponding programs to reduce the time required to process a large number of paper documents. The electronicization of a large amount of data makes the information transfer faster and achieves the purpose of information sharing. The powerful report function also enables the staff to obtain valuable analysing reports for decision-making within minutes, providing support for corporate decision-making.

3) Standardize the business processes of the HR department: the human resource information system covers a series of work modules from recruitment, performance, and compensation to training programs and successor planning. The use of the Internet and personal computers has realized the systemization, modelling, integration and automation of human resource management. Information technology helps to centralize and analyze decentralized human resource information, optimize the process of human resource management, and make the past fuzzy management work procedures clearer, ther eby making the human resource management system more complete, the man- agement organization more reasonable and stable, and management data more scientific and complete. At the same time, the human resource management business process is standardized, and the online business process reporting, approval and summary statistics between subordinate units, departments, and employees have been achieved. Furthermore, management time and geographical restrictions have been broken.

\section{Analysis of the development process of Human Resource Management Information System}

\subsection{Related Technology Introduction of Human Resource Management Information System}

\subsubsection{C/S Architecture}

Client-Server (C / S) Architecture. The C / S Architecture usually adopts a two-layer Architecture. The server is responsible for data management, and the client is responsible for completing interaction tasks with the user. The client connects to the server through the local area network, accepts the user's request, and makes a request to the server through the network to operate the database. The server accepts the client's request, submits the data to the client, and the client calculates the data and presents the result to the user. The server must also provide perfect security protection and processing of data integrity, which allow multiple clients to access the server at the same time, which places high requirements on the server's hardware data processing capabilities. The C / S Architecture is technically very mature. Its main features are strong interactivity, safe access mode, fast response speed, and conducive to processing large amounts of data. However, the C / S Architecture lacks generality. System maintenance and upgrades need to be redesigned and developed, which increases the difficulty of maintenance and management. Further data expansion is more difficult, so the $\mathrm{C} / \mathrm{S}$ Architecture is limited to small LAN. 3.1.2 B/S Architecture

The B / S (Browser / Server) architecture is also known as the browser / server architecture. This architecture can be understood as a change and promotion of the C / S architecture. Due to the rapid development of the network, the functions of the B / S Architecture become more and more powerful. This Architecture can perform distributed information processing, which can effectively reduce resource costs and improve the performance of the designed system. The B / S archi- 
tecture has a wider range of applications and greatly simplifies the client in the processing mode. Users only need to install a browser, and the application logic is concentrated on the server and middleware, which can improve data processing performance. In terms of software versatility, the client of the $\mathrm{B} / \mathrm{S}$ architecture has better versatility and less dependence on the application environment. At the same time, because the client uses a browser, it is more convenient for development and maintenance, which can reduce system development and cost of maintenance. Facing the future, users can access and use the system in different environments through multiple devices such as mobile phones, tablets, and computers.

\subsubsection{Database Technology}

Database technology is a core technology of information systems. It is a method of computer-aided data management. It studies how to organize and store data, and how to efficiently acquire and process data. The object of database technology research and management is data, so the specific contents of database technology mainly include: establish the corresponding database and data warehouse according to the specified structure through the unified organization and management of data; design a data management and data mining application system that can add, modify, delete, process, analyze, understand, report, and print data in the database; and use the application management system to finally implement data processing, analysis and understanding.

\subsection{Introduction to the structure of Human Re- source Management Information System}

The human resource management information system should be divided into three levels from the functional structure: basic data layer, business processing layer and decision support layer. This concept is consistent with Chaojun Liang's view (2010).

1) Basic Data Layer. The basic data layer contains static data and dynamic data with little change. There are two main types of static data, one is employee personal attribute data, such as name, gender, education, etc., the other is enterprise data, such as enterprise organizational structure, job settings, salary levels, management systems, etc. The basic data is used when the HR system is initialized, which is the basis for the normal operation of the entire system. Dynamic data is continuously generated by employees over time after entering the enterprise, such as salary data and assessment data. Dynamic data requires companies to track, identify, collect, and update them in a timely and accurate manner. It is the main source of data for business leaders to grasp the human resource status, management level, and provide future decision support.

2) Business Processing Layer. The business processing layer refers to the system functions corresponding to the specific business processes of human resources management. These functions will continuously generate and accumulate new data in daily management work, such as new employee data, salary data, performance evaluation data, training data, and attendance and vacation data and so on. The manager will realize the functional affairs of human resource management through the query, modification and deletion of these data and information, such as personnel recruitment, training, salary settlement, performance evaluation, etc. This will shorten staff time and greatly improve the level and efficiency of human resource management.

3) Decision Support Layer. The decision support layer is based on an HR database composed of basic data and a large amount of business data, and classifies, statistics and analyzes a large amount of human resource information. When an organization needs to make personnel decisions, it can use the human resource management information system to quickly obtain the required information (such as salary status, employee assessments, etc.) and analyze the results to practice corresponding management activities, which can not only improve the management efficiency of human resources, but also convenient for the senior management of the enterprise to grasp the situation of human resources in general.

\subsection{Analysis of the development process of Human Resource Management Information System}

According to the system life cycle theory, the development process of human resource management informationization can be summarized into four stages: overall planning, system selection, system implementation, and system operation and maintenance. They run through the entire life cycle of HRMIS. The followings are detailed descriptions of each stage.

\subsubsection{Overall Plan}

The overall plan is to formulate a long-term and comprehensive plan in accordance with the development strategy and business objectives of the enterprise, and 


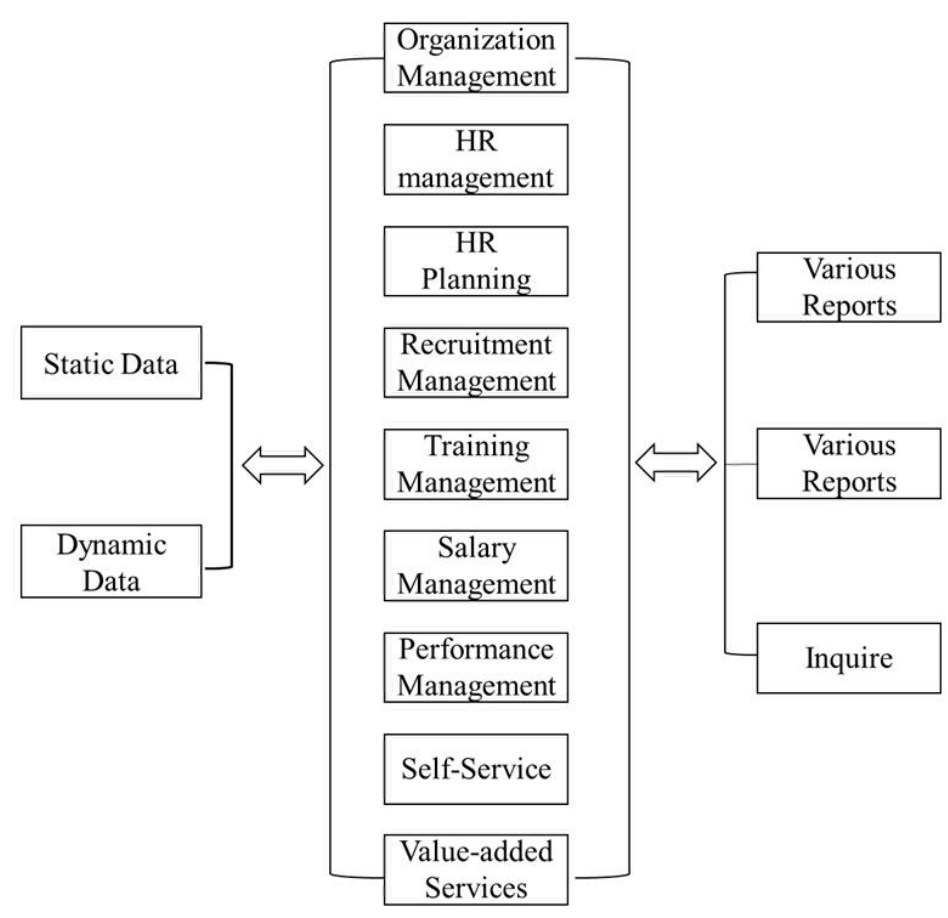

the objective laws of the development of the management information system. The Overall Plan consists of five parts: preparation, analysis, scope of system implementation, target setting, project feasibility analysis.

\section{1) Preparation}

The overall planning of human resource management informatization is actually a comprehensive reflection of the company's development strategy, management philosophy and corporate culture. The development strategy and business direction of the enterprise must be used to influence the direction of human resource management informatization, so that all departments within the company, especially the department leaders, have a clear understanding of the human resource management informatization work, and then in the future project implementation process can get the support and help of various departments to promote the smooth progress of the project. Therefore, when setting up a human resource management informatization team, at least representatives of all functional departments and business departments should be included. Generally, the team of a human resource management informatization project includes the following groups:

a) The senior leadership of the enterprise and the implementer, who are mainly responsible for project planning and supervision.

b) Human resources and information technology personnel, representatives of other functional depart- ments and business departments, implementation consultants and development consultants, etc. They are mainly responsible for the specific implementation of the project.

c) Implementing consultants and third-party consulting agencies, who are mainly responsible for consulting and improving customer business process and management issues in the early stage of the project.

\section{2) Analysis}

This stage mainly analyzes three aspects:

a) Internal and external environmental analysis. From the perspective of the external macro environment, it is necessary to analyze the current development of information technology, including the development of hardware and software, the application of enterprise informatization, and the informationization of the industry and competitors of the enterprise. From the perspective of the internal micro-environment of the enterprise, analyzing the current status of human resource management within the enterprise, such as whether the policy system is sound and the business processes are reasonable, etc. At the same time, it is essential to analyze the current status of enterprise informationization, evaluate the current level of informationization of the enterprise, and determine the gap between the requirements of the development of HRMIS on the level of enterprise informatization and the current level of the enterprise.

b) Informatization level of human resource managers in enterprises. Human resource management informatization is a new human resource management model and also a change in human resource management. The organizational form has changed, and the corresponding personnel also need to change. For a human resource manager, if he or she is only proficient in human resource management as in the past, then he or she is out of date. Information-based human resource management requires that the HR manager not only be an expert in a certain aspect of human resource management activities, but also an owner of comprehensive understanding of the information system.

c) Demands analysis. Analyzing the demands for human resource management informatization in different roles in the enterprise is required. These roles include corporate decision-making layers, human re source managers, business managers of various depart- 
ments, and ordinary employees.

3) Determine the scope of system implementation According to the requirements of human resource management informatization, it is required to conduct a full investigation on the business relationship between the corporate organization and various departments and clarify the institutions and departments that the system needs to involve. Then it is necessary to analyze the functions of each business department, and select the business group directly related to the system requirements as the scope of system research. This range reflects the basic size of the final system and determines the boundaries of the final system.

4) Determine the target

The overall goal of enterprise human resource management informatization is to expect the ultimate function that the enterprise human resource management informatization can achieve and the effect it brings to the enterprise. It is the direction and guidance of system development. In the system planning, the background, purpose and role of the enterprise in the development of human resource management informatization must be clarified. At the same time, it is necessary to work out the development direction of enterprise
The feasibility of human resource management information development projects is generally considered from three aspects: economic feasibility, technical feasibility and social feasibility. Specifically, the feasibility analysis of the implementation of the new system can be carried out by conducting a preliminary survey of the enterprise and combining the initial scheme of the new system.

\subsubsection{System selection}

In this selection, the types of HRMIS and the process of the selection of HRMIS are introduced.

\section{1) The type of HRMIS}

Different enterprises have different staff sizes, organizational structures, and business development models, so their human resource management levels will not be identical. When choosing a HRMIS, not every function of human resources needs to be informatized, nor is the operation process of each module of human resources to be informatized. It is best to choose a system that suits your company's development stage and management characteristics. According to the complexity of human resource management informatization, the following four types are given:

2) Process of selection of HRMIS

\begin{tabular}{c|c|c|c}
\hline Type & Feature & Technical Requirement & Suitable Company \\
\hline Database Type & $\begin{array}{c}\text { Electronic archive management, } \\
\text { human resource information search } \\
\text { and query, Focusing on the } \\
\text { processing of information materials }\end{array}$ & $\begin{array}{c}\text { Stand-alone, General } \\
\text { configuration }\end{array}$ & $\begin{array}{c}\text { Low level of informatization, } \\
\text { Small scale, newly established } \\
\text { enterprises, Lack of funds and HR } \\
\text { talents. }\end{array}$ \\
\hline Independent Function Type & $\begin{array}{c}\text { Single or multiple HR management } \\
\text { function modules, Automated } \\
\text { processing of most HR job preocess }\end{array}$ & $\begin{array}{c}\text { Simple LAN, General } \\
\text { configuration }\end{array}$ & $\begin{array}{c}\text { Existing informationization level } \\
\text { is moderate; Personnel reach a } \\
\text { certain scale; Insufficient funds } \\
\text { and HR talents; Heavy HR } \\
\text { management tasks }\end{array}$ \\
\hline Function Integration & $\begin{array}{c}\text { Complete HR management function } \\
\text { module, Information sharing of each } \\
\text { function module, }\end{array}$ & $\begin{array}{c}\text { C / S, B / S architecture, } \\
\text { Internet access }\end{array}$ & $\begin{array}{c}\text { Existing informatization; Large } \\
\text { staff scale; Sufficient funds and } \\
\text { excellent talents }\end{array}$ \\
\hline System Function Type & $\begin{array}{c}\text { Sharing information with other } \\
\text { management systems, Highly } \\
\text { customized, Providing strategic } \\
\text { forecasting, planning and decision } \\
\text { support, HR in-depth development } \\
\text { support }\end{array}$ & $\begin{array}{c}\text { High-performance servers and } \\
\text { network hardware } \\
\text { Internet access, }\end{array}$ & $\begin{array}{c}\text { High degree of informatization; } \\
\text { Sufficient funds and various } \\
\text { talents }\end{array}$ \\
\hline
\end{tabular}

human resource management informatization to realize the transformation of human resources from a cost center to a value creation center.

5) Project feasibility analysis
When choosing a HRMIS, based on insufficient research and understanding of suppliers and their products, you must not easily choose a system that others prefer, but rather follow a scientific selection method. 
In the process of finding a suitable solution, the enterprise should follow the standard selection process, which can not only help the enterprise avoid various "traps", effectively avoid risks, but also greatly shorten the selection cycle and reduce costs.

The selection of human resource management information system generally goes through the following steps:

a) Setting up a working team

Set up a selection project team composed of people according to the size of the project. In addition to the key personnel of the HR department and the technical staff of the IT department, the team members should also include middle and senior leaders of the enterprise, responsible for approving the budget and participating in contract negotiations.

b) Determining system goals

Before the selection project begins, the system goals must be formulated, and a goal specification must be written. The instructions should include these:

What effect the e-HR system brings to the enterprise;

What is the strategy of the HR department;

What business processes the company wants to change through the system;

How the system provides comprehensive support for HR business needs.

c) Needs analysis

The requirements analysis is mainly to answer what functions the system provides for the enterprise, why they are needed, and whether these functions can achieve these problems with an acceptable price.

d) Clearing system requirements

System requirements include requirements for hardware and software. For example, whether the company's organizational structure and business processes are reasonable; whether it has advanced human resource management concepts and corporate culture; whether the job level is clear; whether the technical environment required by the system is available.

e) Drawing up a project budget

The budget mainly includes three parts: software system costs, implementation costs, and corresponding operating environment development costs.

f) Preparation of requirement statement

The requirement statement mainly informs the supplier of the basic situation of the enterprise and the requirements of the system, which mainly includes the fol- lowing:

Enterprise profile and human resources management status;

Project development goals, implementation scope, number of employees to be supported, number of users, etc .;

Detailed description of functional requirements and performance requirements;

Enterprise's existing infrastructure environment description and technical requirements;

Project schedule and time requirements;

The requirements for supplier prices mainly include software license fees, training implementation fees, and daily maintenance fees;

List of companies that have been implemented by suppliers, contact information, implementation effects, etc.

g) Primary supplier selection

An enterprise can conduct initial selection of suppliers by way of corporate invitations, public bidding, and internal negotiation, and then send project demand specifications to qualified suppliers.

h)Evaluation proposal

After sending the demand statement, the company will receive the proposal submitted by the supplier. The members of the selection project team should evaluate the supplier's plan together with the experts.

i) System demonstration

The system demonstration creates face-to-face communication opportunities for enterprises and suppliers. In the demonstration, companies can ask questions about the status of their human resources management business, and propose some specific human resource management business scenarios, such as staffing, mobility, and salary adjustments, contract changes, etc., to check the applicability of the system.

j) Visiting suppliers and typical customers

Through field inspections, company can further understand the operation status and strength of suppliers; to visit typical customers, especially typical customers in the same industry, company can determine whether the functions of the supplier system meet their needs, and whether the implementation effect is close to expectations.

k) Adjustment plan

Through on-site communication with suppliers and customers, the demand specification is supplemented and adjusted again, and suppliers are required to re- 
spond and confirm the adjustment of the demand plan. 1) Final decision

After the visits to suppliers and customers, the project team makes the final decision to determine the best choice for the supplier.

\subsubsection{System Implementation}

After the current overall plan and system selection are completed, the next step is the project implementation process. This process is based on the human resource management business demands of the enterprise, formulating corresponding solutions to help enterprises successfully apply human resource management information systems to improve management efficiency. The system implementation process is the most critical step in the entire information development process. The quality of the implementation results is directly related to the realization of the company's strategic goals. According to the successful implementation cases and best business practices of domestic and foreign human resource management software vendors, the author believes that the best implementation process should be divided into four steps, as shown in the following figure: ational difficulties caused by process changes, data errors, and system software upgrade issues.

Therefore, after the e-HR system goes online, it is also an important task to formulate various management systems to ensure the normal operation and benefit of the system. System maintenance includes maintenance of application systems, data, code, documents, hardware equipment, and so on. There are three ways to maintain the system:

Establish effective inspection, communication and training mechanisms to ensure that the system settings can meet the changes in relevant corporate policies and meet the requirements of different users.

Regularly clean and back up the system to clean up useless data and improve operating efficiency.

Establish a running log file to effectively record problems during system operation and avoid repeated errors in future use; regularly update system setting instructions and user documentation to help new users better understand system requirements and be familiar with operating procedures and methods, avoid risks, improve system availability and user confidence in the system.

\begin{tabular}{|c|c|}
\hline Preparation for Implementation & Blueprint Design \\
\hline $\begin{array}{l}\text { - Establish implementation organization } \\
\text { - Develop implementation strategies and } \\
\text { plans } \\
\text { - Develop a quality and risk strategy } \\
\text { - kick-off conference }\end{array}$ & $\begin{array}{l}\text { - } \text { Product Manager Training } \\
\text { - Key user training } \\
\text { - } \text { Business research and carding analysis } \\
\text { - } \text { System environment deployment } \\
\text { Explanation of the implementation plan }\end{array}$ \\
\hline
\end{tabular}

\begin{tabular}{|l|}
\hline \multicolumn{1}{|c|}{ Continuous Support } \\
\hline - \\
- Useutine Maintenance Strategy \\
- System operation support \\
- Implementation of effect evaluation \\
\hline
\end{tabular}

\begin{tabular}{|ll|}
\multicolumn{1}{|c|}{ Online Implementing } \\
\hline$\bullet$ & System Configuration \\
$\bullet$ & Data acquisition and import \\
- & Solution design \\
- & Test preparation and grass-roots testing \\
- & System initialization \\
- & Writing user manuals \\
\hline & System switching online \\
\hline
\end{tabular}

3.3.4 System Operation and Maintenance

Many companies believe that after the successful implementation of the HRMIS, the entire project is complete. In fact, this is not the case. The successful launch of the system is just the beginning of enterprise informationization. After the launch, there will be a gradual adaptation process. During this period, various problems will occur, such as failure of key users, oper-

\section{Conclusion}

With the development of the times and the progress of society, human resource management has undergone great changes in terms of ideology, theory, and practice, and it has also been paid more and more attention by the society. The advent of the information age has changed the methods and means of human resource management. With the high-speed transmission of information today, the competition facing enterprises is becoming more and more fierce. Informatization has become a powerful tool for enterprises to improve management efficiency and effectiveness, and HRMIS has also emerged. The continuous development of information 
technology has also provided a guarantee for the continuous improvement and upgrade of the human resource management information system. Human resource management informatization has become an important tool to promote the rapid appreciation of human capital and win competitive advantages. It will also become an important investment area and strategic direction for enterprises in the future. Scientific planning, successful implementation, and effective use are of great significance for improving the management level of an enterprise.

This article briefly introduces the technologies and concepts that may be involved in the article, summarizes the current field and the corresponding theoretical knowledge involved, introduces the process of building a human resource management information system, and analyzes each step-in detail.

This paper has gained valuable practical experience by studying the theory and application of human resource management informatization. The conclusions of this article are summarized as follows:

First, the informationization of human resource management is an inevitable trend in the future development of enterprises and an important sign of the im provement of management level. The change of human resource management methods and changes in work methods will inevitably require enterprises to implement informatization of human resource management.

Second, scientific systematic planning is the prerequisite for successful implementation of e-HR projects. Different enterprises have different levels of human resource management and should combine their own characteristics to achieve correct positioning, global planning, and distributed implementation.

Third, a good informatization foundation and clear management processes are necessary conditions for the implementation of human resource management informatization. Optimizing the management model first, then optimizing the management tools. Clarifying the requirements before determining the system functions is the ideal strategy for the development of human resource management informationization in Chinese enterprises.
Fourthly, when selecting suppliers, we must follow the scientific selection process, combining with our own specific conditions, do not blindly worship so-called authorities.

Fifth, effective communication is vital to the success of the project throughout the entire life cycle of the system development.

Sixth, we must make timely and accurate evaluations of the effects of human resource management informatization, and constantly find and correct problems so that the human resource management information system can maximize its effectiveness.

Seventh, the realization of the ideal effect of human resource management informatization requires the participation of all employees and the establishment of a comprehensive human resource management awareness.

Finally, human resource management informatization is just a new model of human resource management. People cannot rely too much on the system to solve every problem. Otherwise they ignore subjective initiative and ignore their own changes and development.

\section{References:}

[1] Dazheng Zhang (2016). Design and implementation of human resource management information system.

[2] Zhaojun Liang (2010). Research on the Development of Human Resource Management Information System -Based on the perspective of enterprise human resource value promotion.

[3] Wei Xie (2011). Research on the Issues Related to the Informatization of Human Resource Management in China's Enterprises.

[4] Wentao Yuan (2009). Application of Information System in Modern Human Resource Management.

[5] Qian Wang, Chao Pang, Ying Wang (2019). Discussion on the Development and Application of Human Resource Management Information System .

[6] Haifeng Wang, Ping Zhang, Yuanlei Wang, Beibei Li (2019). Application of Information System in Human Resource Management.

[7] Meina Chen, Yan Qin, Yuxin Zhao (2019). An Investigation Report on the Application Status of Enterprise Informatization Human Resource Management Software. 